\title{
An improved method for R-peak detection by using Shannon energy envelope
}

\author{
M RAKSHIT, D PANIGRAHY* and P K SAHU \\ Department of Electrical Engineering, National Institute of Technology Rourkela, Rourkela, Odisha 769008 , \\ India \\ e-mail: rakshitmanas09@gmail.com; damo.3093@gmail.com; pksahu@nitrkl.ac.in
}

MS received 16 December 2014; revised 21 December 2015; accepted 25 December 2015

\begin{abstract}
R-peaks in electrocardiogram (ECG) play a vital role in diagnosis of heart rhythm irregularities and also estimating heart rate variability. However, almost all existing R-peak detectors suffer from the nonstationary of both QRS morphology and noise. To overcome these difficulties, we propose a four-stage improved method to detect R-peak using Shannon energy envelope. In the first stage, noise is suppressed and QRS complex is enhanced by using band pass filter, first order differentiation, and amplitude normalization. In the second stage, Shannon energy envelope is extracted. In the third stage, peak is estimated without considering any threshold amplitude. In the final stage, true R-peaks are detected. Our proposed R-peak detection method is validated using 48 first channel ECG records of the MIT-BIH arrhythmia database with the accuracy of $99.84 \%$, sensitivity of $99.95 \%$ and positive predictability of $99.88 \%$. Our proposed method outperforms other wellknown methods in case of pathological ECG signals.
\end{abstract}

Keywords. Electrocardiogram; MIT-BIH database; QRS complex; Shannon energy envelope.

\section{Introduction}

Electrocardiogram (ECG) is an important biological signal that reflects the electrical activity of heart. It provides a lot of information on the normal and pathological physiology of heart. An ECG signal consists of different parts such as P wave, QRS complex and T wave [1]. As it is a non-stationary signal, it is very difficult to analyse it visually and therefore a computer based ECG analyzer is required. R-peak detection in ECG is a popular method in order to diagnose heart rhythm irregularities and also to estimate heart rate variability (HRV) [2-4].

Various signal processing algorithms have been developed for detecting R-peak in the ECG. Different methods for detecting R-peaks in ECG are Pan-Tompkins method [5], artificial neural network (ANN) [6], heuristic methods [7, 8], wavelet transform (WT) [9-14], Hilbert transform [15], empirical mode of decomposition [16], Shannon energy with Hilbert transform method [17], and Shannon energy envelope [18].

Pan-Tompkins method (PT) [5] based on moving average filter and peak detection is a low complexity algorithm but yields a lower accuracy. Moreover as the peak detection is based on amplitude threshold that degrades accuracy. ANN [6] requires complex rules for detecting R-peak. The

*For correspondence performance of heuristic methods [7, 8] depends on suitable choice of bandwidth of band pass filter (BPF) and also the duration of moving window used for integration. Wavelet based methods [9-11, 13, 14] require selection of suitable mother wavelet and scales to obtain QRS event. Hilbert transform and empirical mode of decomposition give high accuracy but on a limited number of records in MIT database. Shannon energy with Hilbert transform method (SEHT) [17] provides a good accuracy for detecting R-peak. Hilbert transform in SEHT requires high memory and delays making it unsuitable for real time implementation. For a long pause ECG data, SEHT detects a large number of noise peaks. Computation of Hilbert transform in frequency domain requires very long memory buffer for storing samples of ECG data. In 2013, Zhu and Dong [18] have developed an R-peak detection method called PSEE by using only Shannon energy envelope. Again for valid pair peak detection there is a use of amplitude threshold that affects the performance of the algorithm.

In this paper, we have proposed an algorithm based on the Shannon energy envelope which does not require any amplitude threshold for detecting R-peak. It has also overcome the drawbacks of the R-peaks detection techniques based on Shannon energy envelope [18] with a better accuracy. Our proposed method shows an accuracy of $99.84 \%$ evaluated with all the records of MIT-BIH arrhythmia database $[19,20]$. 
In section 2, the four-stage R-peak detection algorithm is described in detail. We introduce the R-peak detection methodology based on the Shannon energy envelope without requiring any amplitude threshold. The sensitivity, positive predictability and accuracy of our proposed algorithm evaluated from MIT-BIH arrhythmia database are explained in section 3. Finally, we discuss and conclude our study in section 4 .

\section{The proposed R-peak detection methodology}

The block diagram of proposed R-peak detection methodology is shown in figure 1. It consists of four stages namely pre-processing, Shannon energy envelope extraction, peak estimation logic and true R-peak detection. In the preprocessing stage noise is suppressed and QRS complex is enhanced by using a BPF, first order differentiation and followed by amplitude normalization. In the Shannon energy envelope extraction stage, smooth Shannon energy envelope is produced by using Shannon energy calculation and a moving average filter. In peak estimation logic stage, the pre-processing of R-peak detection is performed by using first order differentiation, amplitude normalization, squaring, followed by a moving average filter for smoothing. In true R-peak detection stage, the actual location of
$\mathrm{R}$-peak is detected. The detailed discussion of each stage in figure 1 is presented in the following sections.

\subsection{Data pre-processing}

In reality, the pathological ECG signals are corrupted by various noises such as power line interference noise (60 $\mathrm{Hz}$ ), motion artefact due to electrode, skin interface, patient breathing, etc. Moreover, ECG signal contains $\mathrm{P}$ wave, $\mathrm{T}$ wave other than QRS complex which are not of interest in our work. Therefore, we need a filtering system in order to extract QRS complex. From the power spectral analysis of various signal components in ECG signal, we observe that the maximum power density of QRS complex lies between 5 and $20 \mathrm{~Hz}$ [21]. In our proposed method, the ECG is passed through a Chebyshev type I filter with bandwidth of [6 18 ] $\mathrm{Hz}$ for this purpose.

After bandpass filtering the signal, we perform the first order differentiation of the signal to get the slope information. The first order differentiation is nothing but a high pass filter which passes high frequency components (QRS complex) and attenuate lower frequency components ( $\mathrm{P}$ and $\mathrm{T}$ wave). The mathematical implementation of the first order differentiation is shown below:

$$
\mathrm{d}[n]=\mathrm{f}[n+1]-\mathrm{f}[n] .
$$

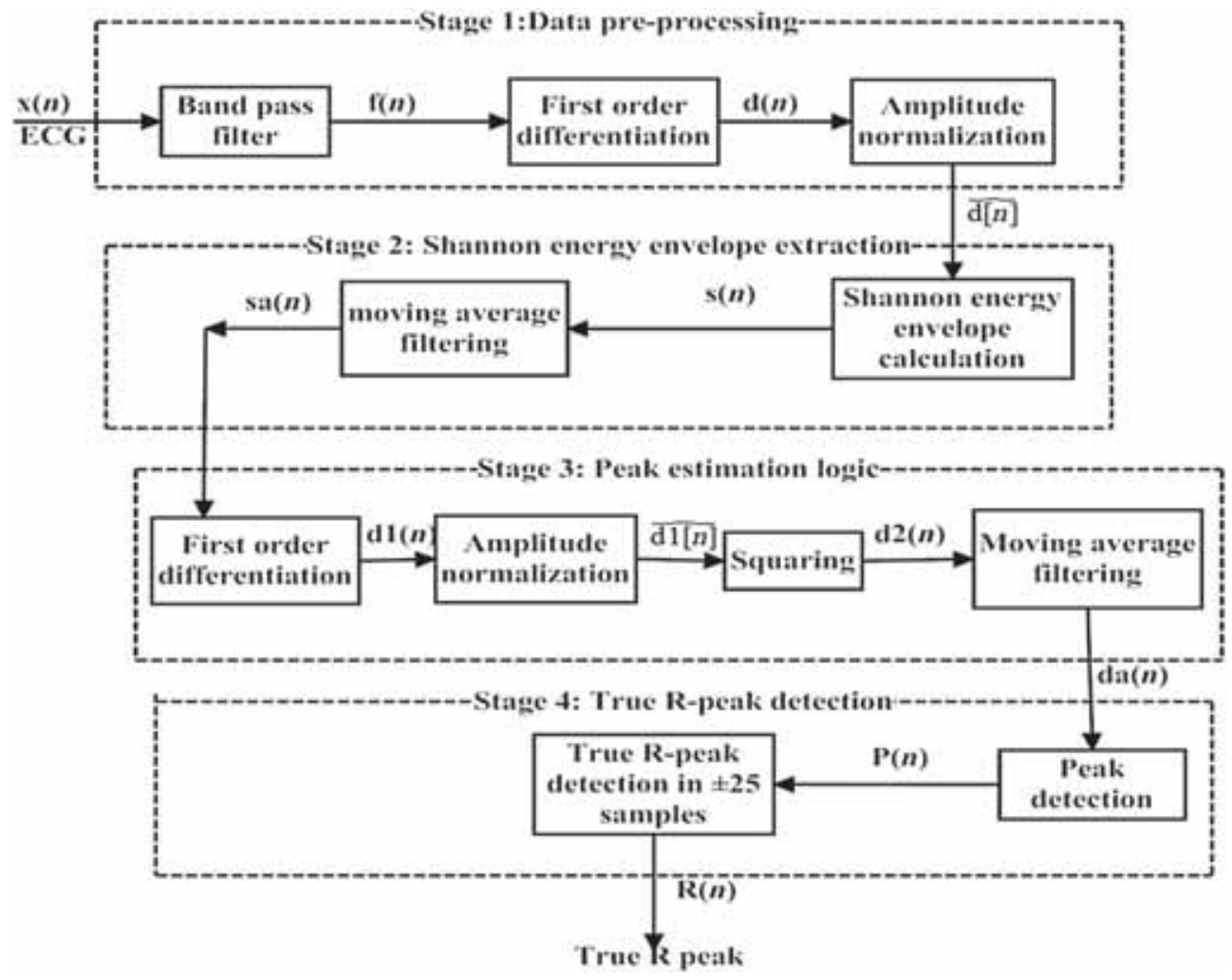

Figure 1. Block diagram of four-stage R-peak detection methodology. 
After filtering and differentiating, the signal is normalized in order to scale its value to 1 . This is done for preparing the signal for Shannon energy envelope computation.

$$
\widehat{\mathrm{d}[n]}=\frac{\mathrm{d}[n]}{\max (|\mathrm{d}[n]|)}
$$

\subsection{Shannon energy envelope extraction}

After differentiating the ECG signal it becomes a bipolar signal. Since the method is based on peak detection, we have to transform the differentiated signal into a unipolar signal. The unipolar signal can be obtained by absolute value method, squared value method, Shannon entropy method, and Shannon energy envelope method. The mathematical formulation of these methods is described below.

$$
\begin{aligned}
& \text { Absolute value: } \mathrm{y}[n]=|\mathrm{a}[n]| \\
& \text { Squared value: } \mathrm{y}[n]=\mathrm{a}[n] \times \mathrm{a}[n] \\
& \text { Shannon entropy value: } \mathrm{y}[n]=-|\mathrm{a}[n]| \times \ln (|\mathrm{a}[n]|) \\
& \text { Shannon energy value: } \mathrm{y}[n]=-(\mathrm{a}[n])^{2} \ln \left((\mathrm{a}[n])^{2}\right)
\end{aligned}
$$

where $\mathrm{a}[n], \mathrm{y}[n]$ are input and output for the above equations respectively.

In the proposed method, we have used Shannon energy envelope method as it has some advantages over the others. Figure 2 shows that absolute value method produces same weight value irrespective of input amplitude. Shannon entropy method attenuates high intensity components and gives high preference to low intensity components. The squared value method gives an exponential weightage response to the high intensity components which will make detection of low intensity components difficult. For Shannon energy method, middle intensity components are more emphasized and other intensity values are attenuated which gives a better detection of R-peaks.

For the sake of getting a unipolar signal, Shannon energy envelope calculation has been employed. When we are taking the logarithm of the normalized derivatives by using the Shannon energy envelope, the maximum amplitude of the normalized derivative is diminished, but not the R-peaks. The absolute value of the amplitude of the normalized derivative corresponds to the R-peaks location lies between 0.4 and 0.8 as shown in figure 3. Shannon energy gives more emphasis when the absolute value of the amplitude lies between 0.4 and 0.8 as shown in figure 2 . The absolute value of the amplitude of normalized derivatives between 0.4 and 0.8 refers to the R-peaks. So to give more emphasize on R-peaks we have used Shannon energy envelope calculation.

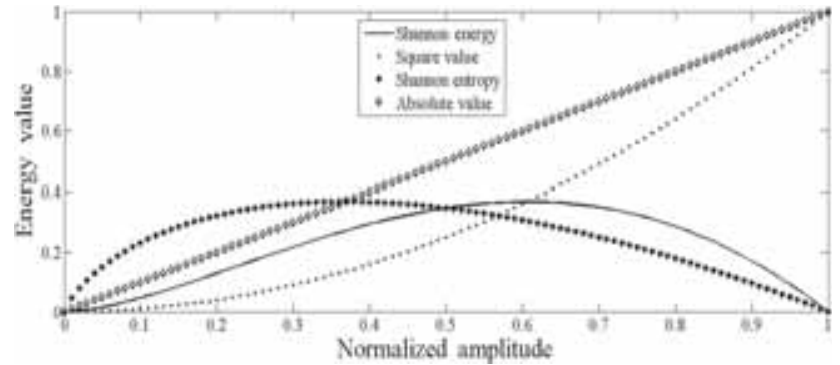

Figure 2. Comparison of energy value for different envelope methods using normalized amplitude.

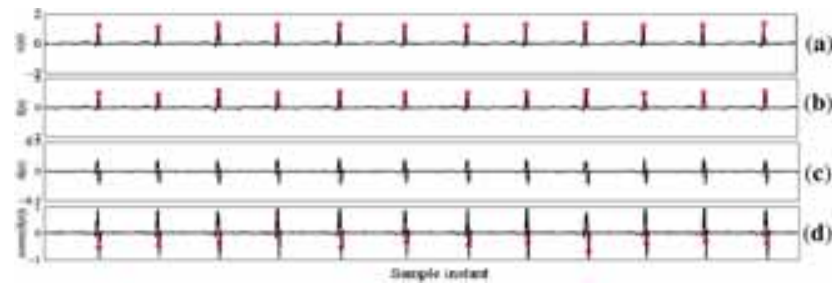

Figure 3. Steps for preparing the signal for Shannon energy envelope calculation.

After extracting Shannon energy envelope, the signal is passed through a zero shift moving average filter in order to get a smooth Shannon energy envelope [17]. The each step of the second stage is shown in figure 4. It shows that after Shannon energy we do not get smooth envelope as shown in figure 4(b), due to its false peaks may be considered as R-peaks. Figure 4(c) shows that a smooth envelope is an output of moving average filter.

A moving average filter is nothing but a moving window integrator. Mathematical expression of a moving average filter is expressed below.

$$
\begin{aligned}
\mathrm{y}(n T)= & \frac{1}{N}[\mathrm{a}(n T-(N-1) T)+\mathrm{a}(n T-(N-2) T) \\
& +\cdots+\mathrm{a}(n T)] .
\end{aligned}
$$

The length of the moving average filter is an important parameter for this detection method. Generally, the length of the moving average filter is taken approximately to the width of the QRS complex. If the length of moving average filter is too large then the integration takes the $T$ wave also, and if the length of the moving average filter is smaller compared to the QRS complex, then for a single QRS complex, it produces more spikes which leads to an erroneous detection. In our proposed method, we have taken a length of 65 samples moving average filter.

The window length of the moving average filter for performing the average operation on the Shannon energy envelope results depend upon the sampling frequency $\left(f_{\mathrm{S}}\right)$ under which the measurements are taken. In this work the window length of the moving average filter for producing smooth envelope is $0.18 \times f_{\mathrm{s}}$. The sampling frequency of 


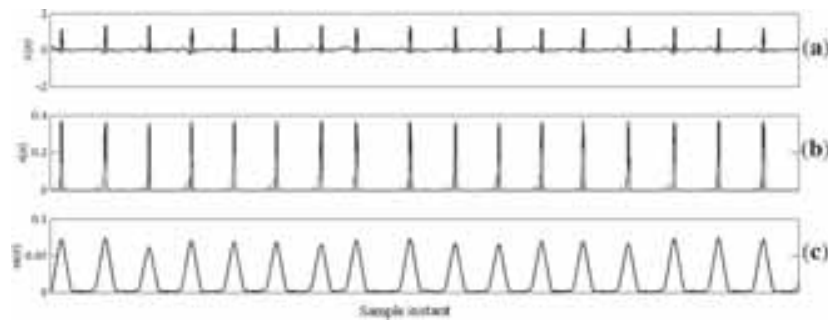

Figure 4. Extraction of smooth Shannon energy envelope.

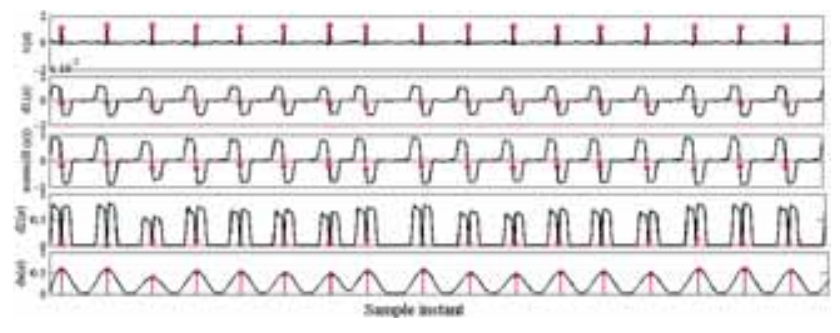

Figure 5. Peak estimation logic without considering any amplitude threshold.

the used database (MIT-BIH arrhythmia database) is 360 . So we have taken the window length of 65 $(360 \times 0.18=64.8 \cong 65)$ samples moving average filter.

\subsection{Peak estimation logic}

In this stage, we introduce a different approach from the other R-peak detection technique based on Shannon energy. Manikandan et al [17] use Hilbert transform with Shannon energy for detection of R-peaks but the Hilbert transform requires more memory buffer. Zhu and Dong [18] use amplitude threshold in the decision stage for extraction for true R-peaks. To overcome these problems, our R-peak detection logic should be free from amplitude threshold. After Shannon energy envelope extraction stage, we get smooth peak signal but it contains both true R-peaks and false R-peaks. In this stage, we actually attenuate the false R-peaks and emphasize the true R-peaks. This is done by following steps.

It is natural that the amplitude value for true R-peaks will be quite high than the false peaks. So if we take the first order differentiation of the signal, then it stores the slope information of the true peaks but it reduces the slope information of the false peaks.

First the signal is amplitude normalized to unity. The normalized bipolar signal is converted to unipolar signal by squaring operation. The amplitude of false peaks is very low and the squaring operation will attenuate these peaks completely. As a result, the true R-peaks are amplified and false R-peaks are diminished.

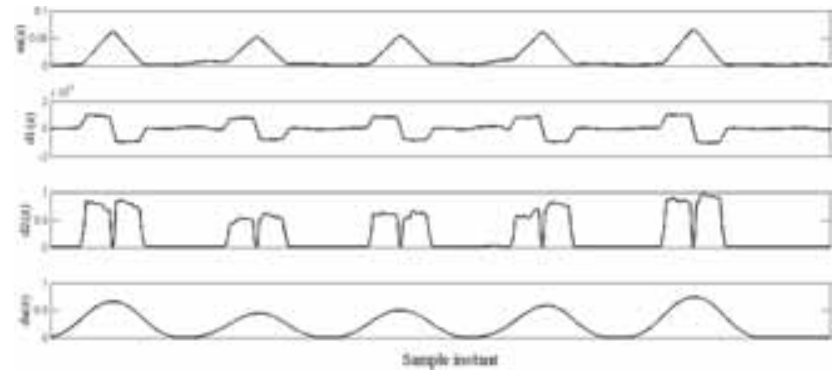

Figure 6. Different signal processing steps of peak estimation logic stage.

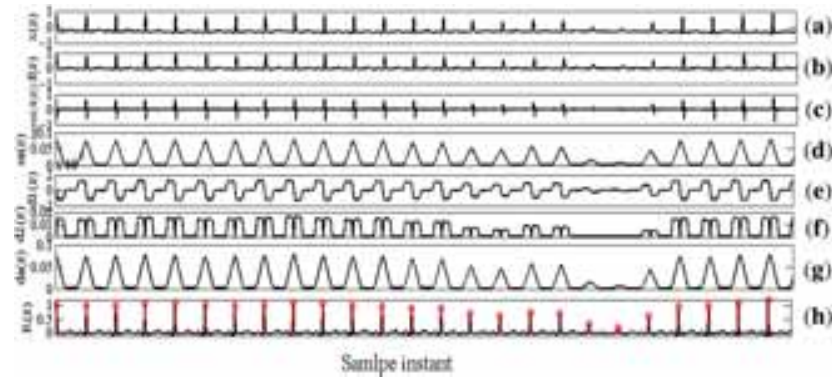

Figure 7. Total signal processing steps of the proposed R-peak detector: (a) ECG signal (Record 205). (b) Filtered signal. (c) Output of normalize operation. (d) Smooth Shannon energy envelope. (e) Differentiation of Shannon energy envelope. (f) Output of square operation. (g) Output of moving average filter. (h) True detected R-peaks (red colour impulses).

Then, the signal is passed through a moving average filter in order to get a smooth peak signal. In our proposed technique, we use a moving average filter of length 85 samples. Now signal is ready for peak detection.

Figure 5 shows labelled diagram of peak estimation logic without considering any amplitude threshold. After squaring, the smooth signal cannot be achieved, to get smooth signal moving average filter operation is performed. The output of the moving average filter does not provide any unnecessary rising peaks. So no threshold operation is required. The locations of rising peaks are referred to the locations of true R-peaks as shown in last subplot of figure 5. Thus, no amplitude threshold value is required for detection of R-peaks.

This peak estimation logic stage is demonstrated in figure 6 .

\subsection{True R-peak detection}

In this stage, the R-peaks are detected by applying peak finding algorithm. However, the detected peak locations are slightly different from the actual positions of the R-peaks in 
Table 1. Performance of our proposed R-peak detection method using MIT-BIH arrhythmia database with length 30 min.

\begin{tabular}{|c|c|c|c|c|c|c|c|}
\hline Data no. & $\mathrm{TP}$ & $\mathrm{FN}$ & FP & $\mathrm{Se}(\%)$ & $+\mathrm{P}(\%)$ & DER $(\%)$ & $\operatorname{Acc}(\%)$ \\
\hline 100 & 2272 & 1 & 0 & 99.95601 & 100 & 0.044014 & 99.95601 \\
\hline 101 & 1866 & 0 & 2 & 100 & 99.89293 & 0.107181 & 99.89293 \\
\hline 102 & 2187 & 0 & 0 & 100 & 100 & 0 & 100 \\
\hline 103 & 2084 & 0 & 0 & 100 & 100 & 0 & 100 \\
\hline 104 & 2255 & 0 & 4 & 100 & 99.82293 & 0.177384 & 99.82293 \\
\hline 105 & 2581 & 5 & 5 & 99.80665 & 99.80665 & 0.387447 & 99.61405 \\
\hline 106 & 2000 & 3 & 5 & 99.85022 & 99.75062 & 0.4 & 99.60159 \\
\hline 107 & 2136 & 1 & 0 & 99.95321 & 100 & 0.046816 & 99.95321 \\
\hline 108 & 1762 & 5 & 8 & 99.71703 & 99.54802 & 0.737798 & 99.26761 \\
\hline 109 & 2532 & 0 & 3 & 100 & 99.88166 & 0.118483 & 99.88166 \\
\hline 111 & 2124 & 0 & 4 & 100 & 99.81203 & 0.188324 & 99.81203 \\
\hline 112 & 2539 & 0 & 1 & 100 & 99.96063 & 0.039386 & 99.96063 \\
\hline 113 & 1795 & 0 & 0 & 100 & 100 & 0 & 100 \\
\hline 114 & 1879 & 0 & 4 & 100 & 99.78757 & 0.212879 & 99.78757 \\
\hline 115 & 1954 & 0 & 6 & 100 & 99.69388 & 0.307062 & 99.69388 \\
\hline 116 & 2405 & 5 & 1 & 99.79253 & 99.95844 & 0.24948 & 99.75114 \\
\hline 117 & 1535 & 0 & 1 & 100 & 99.9349 & 0.065147 & 99.9349 \\
\hline 118 & 2276 & 2 & 5 & 99.9122 & 99.7808 & 0.307557 & 99.69339 \\
\hline 119 & 1985 & 2 & 4 & 99.89935 & 99.79889 & 0.302267 & 99.69864 \\
\hline 121 & 1866 & 0 & 4 & 100 & 99.7861 & 0.214362 & 99.7861 \\
\hline 122 & 2476 & 0 & 0 & 100 & 100 & 0 & 100 \\
\hline 123 & 1520 & 0 & 5 & 100 & 99.67213 & 0.328947 & 99.67213 \\
\hline 124 & 1628 & 0 & 8 & 100 & 99.511 & 0.4914 & 99.511 \\
\hline 200 & 2610 & 0 & 4 & 100 & 99.84698 & 0.153257 & 99.84698 \\
\hline 201 & 1962 & 1 & 2 & 99.94906 & 99.89817 & 0.152905 & 99.84733 \\
\hline 202 & 2136 & 0 & 2 & 100 & 99.90645 & 0.093633 & 99.90645 \\
\hline 203 & 2981 & 9 & 8 & 99.699 & 99.73235 & 0.570278 & 99.43296 \\
\hline 205 & 2655 & 1 & 0 & 99.96235 & 100 & 0.037665 & 99.96235 \\
\hline 207 & 1857 & 3 & 5 & 99.83871 & 99.73147 & 0.430802 & 99.57105 \\
\hline 208 & 2948 & 7 & 3 & 99.76311 & 99.89834 & 0.339213 & 99.66193 \\
\hline 209 & 3003 & 2 & 0 & 99.93344 & 100 & 0.0666 & 99.93344 \\
\hline 210 & 2633 & 1 & 2 & 99.96203 & 99.9241 & 0.113938 & 99.88619 \\
\hline 212 & 2748 & 0 & 0 & 100 & 100 & 0 & 100 \\
\hline 213 & 3250 & 1 & 2 & 99.96924 & 99.9385 & 0.092308 & 99.90778 \\
\hline 214 & 2264 & 0 & 2 & 100 & 99.91174 & 0.088339 & 99.91174 \\
\hline 215 & 3362 & 1 & 0 & 99.97026 & 100 & 0.029744 & 99.97026 \\
\hline 217 & 2208 & 0 & 1 & 100 & 99.95473 & 0.04529 & 99.95473 \\
\hline 219 & 2154 & 0 & 0 & 100 & 100 & 0 & 100 \\
\hline 220 & 2048 & 0 & 0 & 100 & 100 & 0 & 100 \\
\hline 221 & 2427 & 0 & 1 & 100 & 99.95881 & 0.041203 & 99.95881 \\
\hline 222 & 2482 & 0 & 1 & 100 & 99.95973 & 0.04029 & 99.95973 \\
\hline 223 & 2606 & 0 & 0 & 100 & 100 & 0 & 100 \\
\hline 228 & 2053 & 0 & 5 & 100 & 99.75705 & 0.243546 & 99.75705 \\
\hline 230 & 2256 & 0 & 2 & 100 & 99.91143 & 0.088652 & 99.91143 \\
\hline 231 & 1571 & 0 & 0 & 100 & 100 & 0 & 100 \\
\hline 232 & 1780 & 0 & 4 & 100 & 99.77578 & 0.224719 & 99.77578 \\
\hline 233 & 3070 & 8 & 2 & 99.74009 & 99.9349 & 0.325733 & 99.67532 \\
\hline 234 & 2753 & 0 & 0 & 100 & 100 & 0 & 100 \\
\hline Total & 109,474 & 58 & 116 & 99.95155 & 99.88416 & 0.164668 & 99.84131 \\
\hline
\end{tabular}

ECG signal. So in order to find the real positions of R-peaks, the actual sample instant of R-peaks in the ECG signal is found by searching the maximum amplitude within \pm 25 samples of the identified location in the previous step.
In figure 7 , the total signal processing of the proposed method is shown, where in figure 7(h) red impulses indicate the detected R-peaks from the proposed method. 
Table 2. Comparison of performance of our proposed method with other methods for detecting R-peaks using MIT BIH database.

\begin{tabular}{|c|c|c|c|c|c|c|c|c|}
\hline Method & $\begin{array}{l}\text { Total beats } \\
(\mathrm{TP}+\mathrm{FN})\end{array}$ & FN (beats) & FP (beats) & $\operatorname{Se}(\%)$ & $+\mathrm{P}(\%)$ & $\operatorname{DER}(\%)$ & $\operatorname{Acc}(\%)$ & $\begin{array}{c}\text { Standard deviation } \\
\text { of Acc }(\%)\end{array}$ \\
\hline Our proposed method & 109,532 & 58 & 116 & 99.95155 & 99.88416 & 0.164668 & 99.84131 & 17.1 \\
\hline PSEE [18] & 109,494 & 93 & 91 & 99.92 & 99.92 & 0.168 & 99.832 & 28.7 \\
\hline WT [12] & 104,184 & 65 & 112 & 99.94 & 99.89 & 0.170 & 99.830 & 31.6 \\
\hline SEHT [17] & 109,496 & 79 & 140 & 99.93 & 99.87 & 0.200 & 99.800 & 29.7 \\
\hline DOM [4] & 109,809 & 58 & 166 & 99.95 & 99.85 & 0.204 & 99.796 & 25 \\
\hline WT [13] & 109,428 & 220 & 153 & 99.80 & 99.86 & 0.340 & 99.660 & - \\
\hline WT [10] & 110,159 & 322 & 120 & 99.89 & 99.70 & 0.402 & 99.599 & 56.5 \\
\hline PT [5] & 109,809 & 507 & 277 & 99.54 & 99.75 & 0.712 & 99.288 & 20 \\
\hline
\end{tabular}

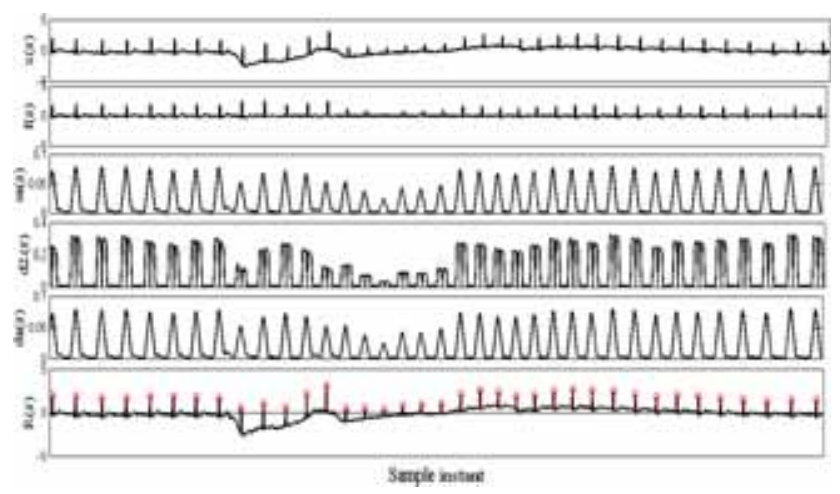

Figure 8. Performance of the proposed method for Record 103 (Low amplitude QRS complexes and baseline drift).

\section{Results and discussion}

Performance of the proposed R-peak detection method is evaluated by using MIT-BIH arrhythmia database [19, 20]. The MIT-BIH Arrhythmia database contains 48 half-hour two-channel ECG recordings, obtained from 47 subjects studied by the BIH Arrhythmia Laboratory between 1975 and 1979. The sampling frequency of the digitized ECG signal is 360 samples per second per channel with 11-bit resolution over a $10 \mathrm{mV}$ range.

The proposed method is implemented on MATLAB R 2012A.

To evaluate the performance of our proposed R-peak detection method, we require three parameters namely true positive (TP), false negative (FN) and false positive (FP)

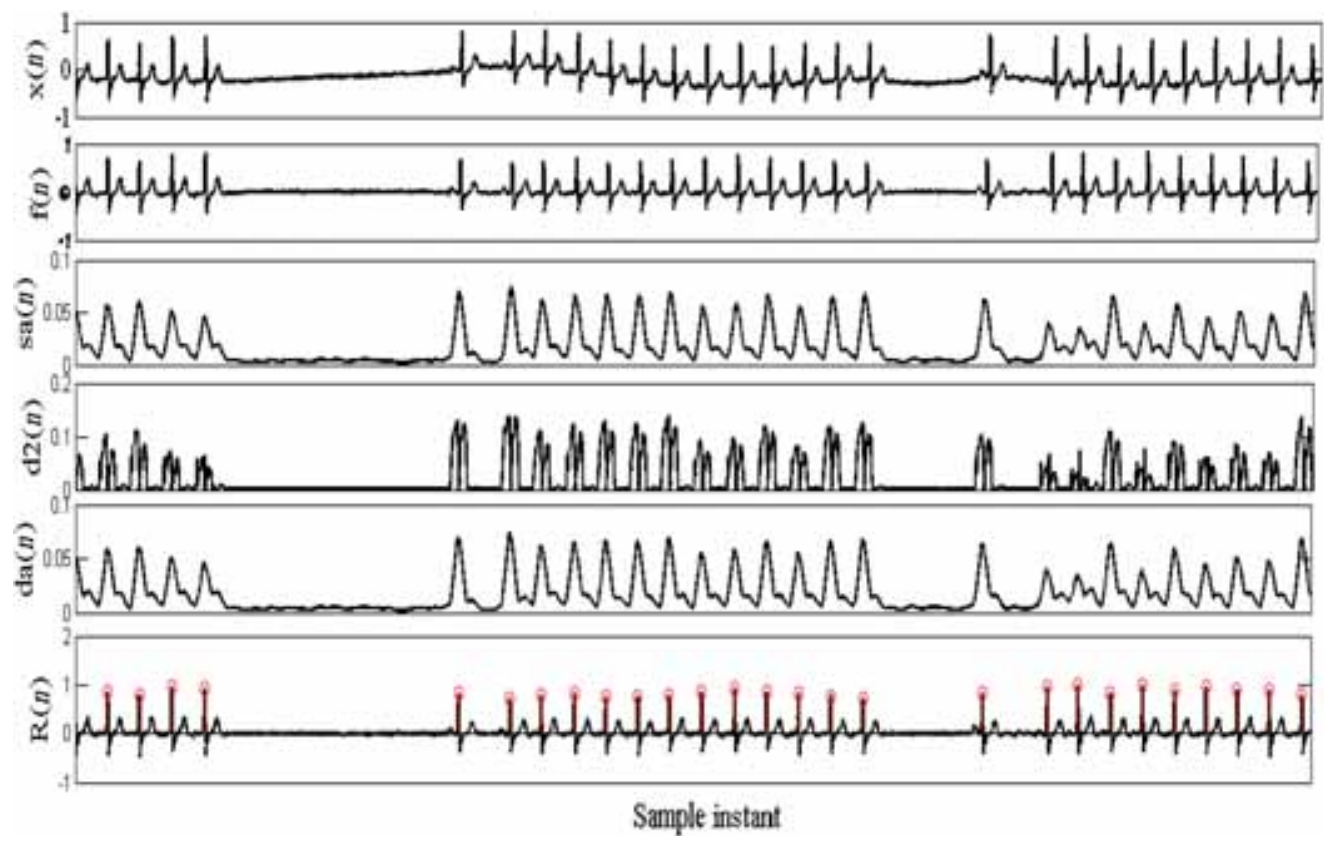

Figure 9. Performance of the proposed method for Record 232 (long pauses). 


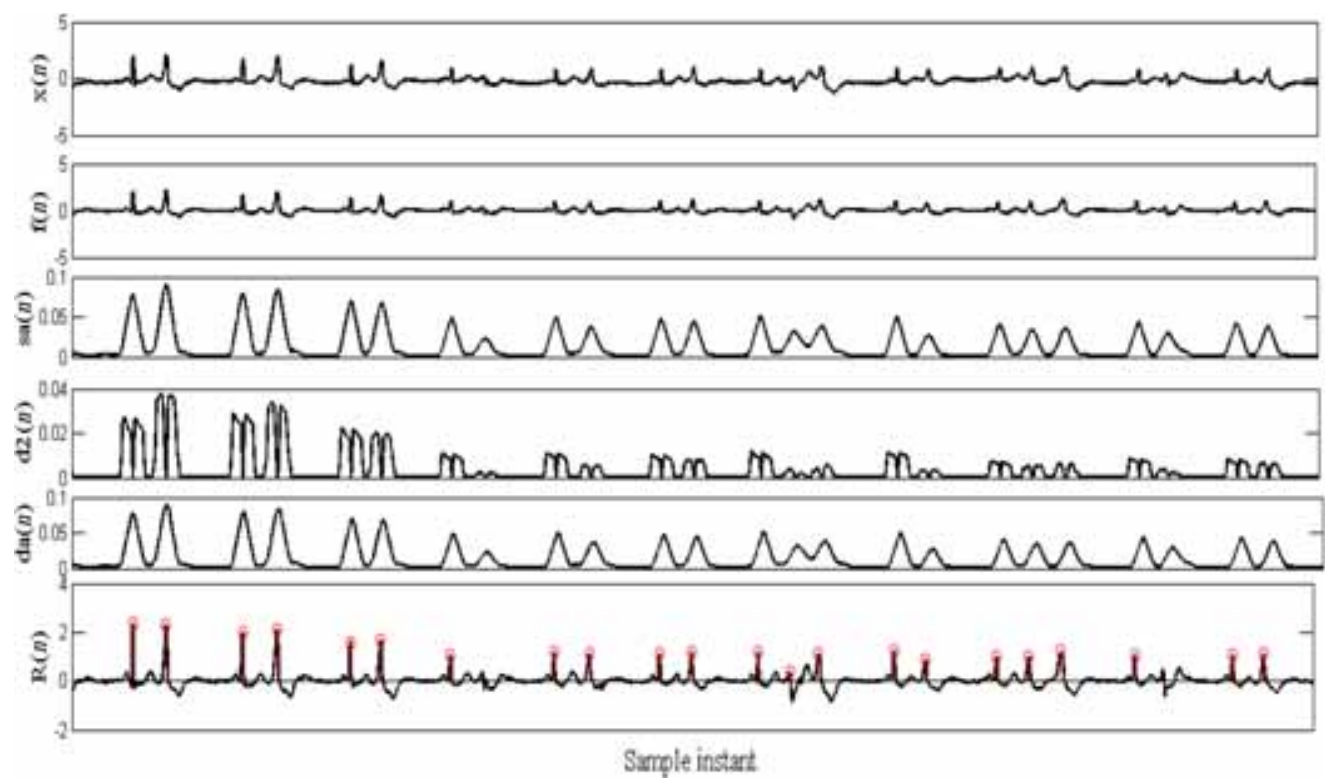

Figure 10. Performance of the proposed method for Record 106 (continuously varying QRS complexes and tall T waves).
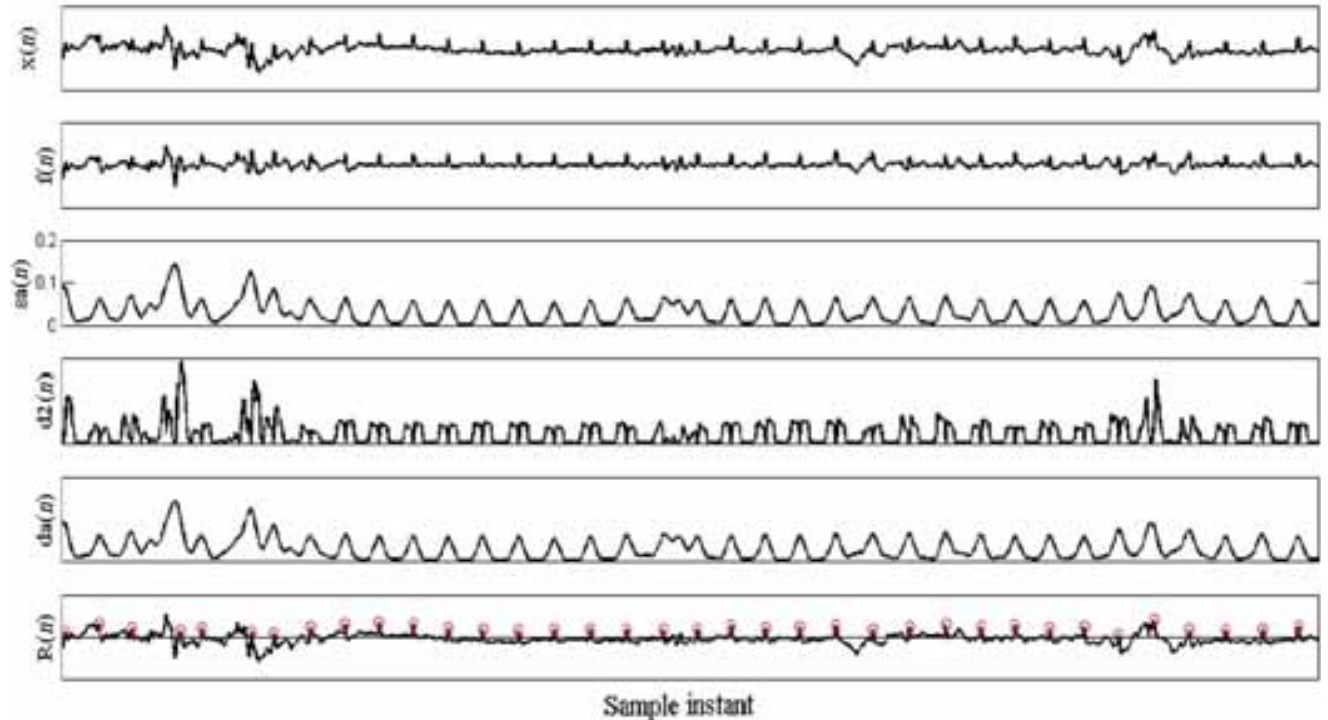

Figure 11. Performance of the proposed method for Record 105 (noisy ECG signal).

from detected R-peak. Here TP is the number of correctly detected R-peaks, FN is the number of missed R-peaks. FP is the number of noise spikes detected as R-peaks. Sensitivity (Se), positive predictability $(+\mathrm{P})$, detection error rate (DER) and accuracy (ACC) can be computed by using TP, FN, and FP by using the following equations respectively.

$$
\mathrm{Se}=\frac{\mathrm{TP}}{\mathrm{TP}+\mathrm{FN}} \times 100 \%
$$

$$
\begin{gathered}
+\mathrm{P}=\frac{\mathrm{TP}}{\mathrm{TP}+\mathrm{FP}} \times 100 \% \\
\mathrm{DER}=\frac{\mathrm{FP}+\mathrm{FN}}{\mathrm{TP}} \times 100 \% \\
\mathrm{Acc}=\frac{\mathrm{TP}}{\mathrm{TP}+\mathrm{FP}+\mathrm{FN}} \times 100 \%
\end{gathered}
$$

The performance of the proposed R-peak detection method for 48 ECG recording of the MIT-BIH arrhythmia database is summarized in table 1. The 


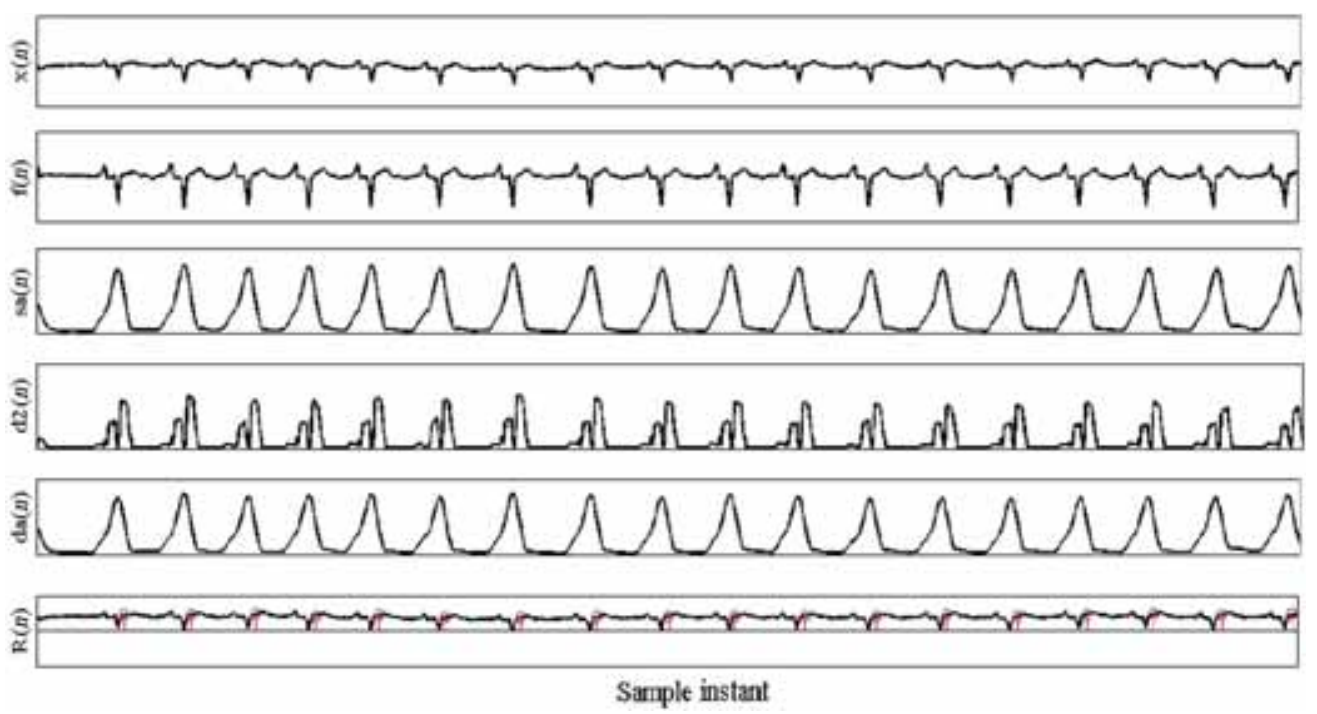

Figure 12. Performance of the proposed method for Record 108 (small R-peaks).

proposed method detects a total number of 109,474 true peaks. It also produces 58 false negative (FN) and 116 false positive (FP). The average accuracy for the proposed method is $99.841 \%$ while the individual detection accuracies vary from $99.26 \%$ to $100 \%$.

In table 2, the performance of the proposed method on MIT-BIH arrhythmia database is compared with other existing methods. It shows that our proposed method has a better accuracy than the other methods like wavelet transform techniques, differential operation method, PanTompkins algorithm, Shannon energy with Hilbert transform technique, Shannon energy technique and our proposed method has less standard deviation compared to other methods.

In MIT-BIH arrhythmia database, records 104, 108, 203, and 228 contain high-grade of noise. Therefore, the accuracy of the method for these records is comparatively weaker than other records. Record 103 contains low amplitude QRS complexes and baseline drift, the performance of the proposed method for this record is shown in figure 8.

Record 232 contains a number of long pauses in the ECG signal. Our proposed method has better response to this signal shown in figure 9.

Record 106 has tall T waves and it contains continuously varying QRS complexes. Detection performance of the expressed method for this signal is shown in figure 10 .

Record 105 has noisy ECG signal. Detection performance of the expressed method for this signal is shown in figure 11 .

Record 108 has small R-peaks ECG signal. Detection performance of the expressed method for this signal is shown in figure 12 .

\section{Conclusion}

In this paper, a computer based automated R-peaks in an ECG signal detection method have been described. This method is a four-stage detection method which is based on the Shannon energy envelope. The detection process of the method is based on band pass filter, first order differentiation, amplitude normalization, Shannon energy envelope, zero shift moving average filter, and squaring. The decision stage is based on first order differentiation, amplitude normalization, squaring and moving average filtering. In this method, the peak finding logic has been improved in such a way that there is no such use of amplitude threshold and no need of prior knowledge of the past detected R-peaks.

In order to evaluate the performance and effectiveness of the proposed R-peak detection method, we have used standard MIT-BIH arrhythmia database which consists of 48 ECG records of $30 \mathrm{~min}$ each. The detection performance of the proposed methodology is evaluated in terms of sensitivity (Se \%), positive predictability $(+\mathrm{P} \%)$, detection error rate (DER \%), and accuracy (Acc \%) which are calculated from true peak (TP), false negative $(\mathrm{FN})$, and false positive (FP). Our proposed method achieves an average accuracy of $99.84 \%$, an average sensitivity of $99.95 \%$ and positive predictability of $99.88 \%$ by using MIT-BIH arrhythmia database. For being a simple mathematical operation based algorithm, the proposed method has a very less computation time which is suitable for real time application. Moreover for ECG signals, containing noise and artefacts like varying QRS morphology, low amplitude, long pauses our proposed method gives a better response than the existing methods. 


\section{References}

[1] McSharry Patrick E, Clifford Gari D, Tarassenko Lionel and Smith Leonard A 2003 A dynamical model for generating synthetic electrocardiogram signals. IEEE Trans. Bio-med. Eng. 50: 289-294. doi:10.1109/TBME.2003.808805

[2] Saini Indu, Singh Dilbag and Khosla Arun 2013 QRS detection using K-Nearest Neighbor algorithm (KNN) and evaluation on standard ECG databases. J. Adv. Res. 4: 331-344. doi:10.1016/j.jare.2012.05.007

[3] Sörnmo L, Laguna P, The Emg, Richard Caton, Hans Berger, Samson T Jacob, Janardhan K Reddy et al 2014 Bioelectrical signal processing in cardiac and neurological applications. Gene Expression 16: 49. doi:10.1002/9780470511923

[4] Yeh Yun C and Wang Wen June 2008 QRS complexes detection for ECG signal: The difference operation method. Comput. Methods Programs Biomed. 91: 245-254. doi:10. 1016/j.cmpb.2008.04.006

[5] Pan J and Tompkins W J 1985 A real-time QRS detection algorithm. IEEE Trans. Bio-med. Eng. 32: 230-236. doi:10. 1109/TBME.1985.325532

[6] Silipo Rosaria and Marches Carlo 1998 Artificial neural networks for automatic ECG analysis. IEEE Trans. Signal Process. 46: 1417-1425. doi:10.1109/78.668803

[7] Hamilton P S and Tompkins W J 1986 Quantitative investigation of QRS detection rules using the MIT/BIH arrhythmia database. IEEE Trans. Bio-med. Eng. 33: 1157-1165

[8] Christov Ivaylo I 2004 Real time electrocardiogram QRS detection using combined adaptive threshold. Biomed. Eng. Online 3: 28. doi:10.1186/1475-925X-3-28

[9] Daamouche Abdelhamid, Hamami Latifa, Alajlan Naif and Melgani Farid 2012 A wavelet optimization approach for ECG signal classification. Biomed. Signal Process. Control 7: 342-349. doi:10.1016/j.bspc.2011.07.001

[10] Ghaffarl A, Golbayani H and Ghasemi M 2008 A new mathematical based QRS detector using continuous wavelet transform. Comput. Electr. Eng. 34: 81-91. doi:10.1016/j. compeleceng.2007.10.005

[11] Legarreta I Romero, Addison P S, Grubb N, Clegg G R, Robertson C E, Fox K A A and Watson J N 2003 R-wave detection using continuous wavelet modulus maxima.
Comput. Cardiol., IEEE, pp 565-568, Conference date: 21-24 September 2003. doi:10.1109/CIC.2003.1291218

[12] Li C, Zheng C and C Tai 1995 Detection of ECG characteristic points using wavelet transforms. IEEE Trans. Biomed. Eng. 42: 21-28. doi:10.1109/10.362922

[13] Martinez Juan Pablo, Almeida Rute, Olmos Salvador, Rocha Ana Paula and Laguna Pablo 2004 A wavelet-based ECG delineator evaluation on standard databases. IEEE Trans. Biomed. Eng. 51: 570-581. doi:10.1109/TBME.2003.821031

[14] Zhang Hongjun 2012 An improved QRS wave group detection algorithm and matlab implementation. In: Phys. Proced., 25: 1010-1016. Elsevier Srl. doi:10.1016/j.phpro.2012.03.192

[15] Benitez D, Gaydecki P A, Zaidi A and Fitzpatrick A P 2001 The use of the Hilbert transform in ECG signal analysis. Comput. Biol. Med. 31: 399-406. doi:10.1016/S0010-4825(01)00009-9

[16] Pal Saurabh and Mitra Madhuchhanda 2012 Empirical mode decomposition based ECG enhancement and QRS detection. Comput. Biol. Med. 42: 83-92. doi:10.1016/j.compbiomed. 2011.10.012

[17] Manikandan M Sabarimalai and K P Soman 2012 A novel method for detecting R-peaks in electrocardiogram (ECG) signal. Biomed. Signal Process. Control 7: 118-128. doi:10. 1016/j.bspc.2011.03.004

[18] Zhu Honghai and Dong Jun (2013) An R-peak detection method based on peaks of Shannon energy envelope. Biomed. Signal Process. Control 8: 466-474. doi:10.1016/j.bspc.2013.01.001

[19] Goldberger A L, Amaral L A, Glass L, Hausdorff J M, Ivanov P C, Mark R G, Mietus J E, Moody G B, Peng C K and Stanley H E 2000 PhysioBank, PhysioToolkit, and PhysioNet: Components of a new research resource for complex physiologic signals. Circulation 101: E215-E220. doi:10.1161/01.CIR.101.23.e215

[20] Moody G B and Mark R G 2001 The impact of the MIT-BIH arrhythmia database. IEEE Eng. Med. Biol. Mag 3: 45-50. doi:10.1109/51.932724

[21] Thakor N V, Webster J G and Tompkins W J 1984 Estimation of QRS complex power spectra for design of a QRS filter. IEEE Trans. Bio-med. Eng. 31: 702-706. doi:10.1109/ TBME.1984.325393 